cluded another new species of Ceratoisis and five other forms previously described. Prof. Hickson and $\mathrm{Mr}$. F. H. Graveley deal with the hydroid zoophytes, which include some interesting forms, especially Hydractinia dendritica, n.sp. Though there is no definitely new generic type, there are ten certainly new species and five more probably new--a very large proportion out of a total of twenty-five. It may be noted that only two of the twenty-five were got outside the limits of McMurdo Bay and the edge of the great ice-barrier, so that we have here a fine representation of the hydroid fauna from the most southerly limit of our knowledge of marine zoology. It is also interesting to find that three of the species are common on British coasts. Dr. John Rennie makes a note on the extraordinarily long tentacles of some unknown siphonophore. They were about as stout as an ordinary bootlace and nearly twenty feet in length. Mr. Hodgson gives a graphic account of the difficulties attending their capture.

Among the sponges, Mr. R. Kirkpatrick found four species of Tetractinellids, forty-three Monaxonellids, twenry-four Calcarea, no Keratosa, and ten species of Hexactinellids. He describes the Hexactinellids, of which three were new genera and eight new species.

The third volume ends with a report on the marine algæ (Pheophyceæ and Florideæ) by Mr. Gepp and Mrs. Gepp, a description of a new coralline by Dr. M. Foslie, and an account of the mosses by M. Jules Cardot. It need hardly be said that with such bulky volumes before us it has not been possible to give more than a hint of the amount of sound and interesting work which they contain.

\section{THE CURE AND PREVENTION OF SLEEPING SICKNESS.}

THE sleeping sickness is, and unfortunately continues to be, the most burning problem of European colonisation in equatorial Africa. Like any other medical problem, that of sleeping sickness has two sides, which may be distinguished broadly as prevention and cure. Investigators in all parts of the world have been experimenting actively with the object of finding a drug, or method of treatment, which shall act in sleeping sickness as quinine does in malaria; that is to say, which shall destroy the parasites in the blood, without seriously affecting the health of the patient. Up to the present, the atoxyl treatment has given the best results, but it has often failed to produce more than temporary amelioration, and it is open to doubt if it has produced a complete cure in any case, while, like other arsenical compounds, it may have serious toxic effects. On Thursday last, however, a communication was made to the Royal Society by Drs. H. G. Plimmer and J. D. Thomson, of the Lister Institute, on the effect of certain antimony salts; and, to judge from the preliminary experiments on rats, these compounds appear to be far more efficient in their curative action, and at the same time less toxic in their effects, than atoxyl. The experiments will be extended at once to larger animals and to man, and though it would be premature to say that the long-sought-for cure has been found, the outlook is certainly more full of hope than it has ever been before.

The question of the prevention of sleeping sickness is of course, bound up with the etiology of the disease. It is known that the disease is caused by the presence of a minute flagellate parasite or "trypanosome," first in the blood, later in the cerebrospinal fluid of the patients; and it is known that the trypanosomes are conveyed from diseased to healthy subjects by the bite of one, possibly more than one, of the species of blood-sucking tsetse-flies. It cannot be too emphatically stated, however, that the tsetse-fly is not, as so often stated, the "cause " of the disease; if the fly be not infected, its bite is harmless, and Koch and others have reported the existence of large areas in which the fly swarms, but in which sleeping sickness does not as yet exist, although the necessary condition for its diffusion is found.

It follows that the problem of prevention may be attacked in two ways; extirpation of the fly, or control of the infection. Considering the vast extent of the range of the species of tsetse-flies in Africa, considering, further, that these flies, being viviparous, have no free larval stages in which they can be destroyed, like mosquitoes, any notion of extirpating tsetse-flies must be considered as frankly utopian. The measures adopted by our Government are wisely directed towards controlling the spread of the infection. Since the fly haunts thick bush on the lakeshore exclusively, the jungle is to be destroyed at all ports, ferries, and landing-places on the lake, where it is unavoidable that human beings should visit the lake-shore; at other points the natives are to be removed from the shore, and persuaded or coerced to live out of the effective range of the fly. Natives known to be diseased are to be segregated, prevented from wandering into the "fly-belts," and placed under treatment. By this means it may be reasonably expected that the spread of the infection may be checked.

There remains, however, the possibility that some wild animal may play a part in spreading the infection, since other animals besides man are known to be susceptible to the trypanosome when inoculated with it in the laboratory. As yet, however, no vertebrate, other than the human species, has been proved to harbour the trypanosome of sleeping sickness in a state of nature. It is well known, however, that other species of trypanosomes, in no way connected with sleeping sickness, are found commonly in wild animals of all classes; and it may be added that the tsetse-flies are quite as willing to suck the blood of a reptile or bird as that of a mammal. Hence there is always the possibility that some species of wild animal may act as a " reservoir" from which the supply of the trypanosome of sleeping sickness may be kept up indefinitely through the agency of tsetse-flies. It is, therefore, of the utmost importance that further researches on the etiology of sleeping sickness should be carried on, with the special object, among others, of discovering any such indigenous source of the disease, for it need hardly be pointed out that it would be of little use to prevent tsetse-flies becoming infected from human beings if they could also obtain the infection from natural sources.

\section{THE POLLUTION OF RIVERS.}

O Thursday, October 3I, an influential deputation from the British Science Guild interviewed Mr. Burns, M.P., at the Local Government Board, upon the subject of legislation with respect to the prevention of the pollution of rivers, and the protection of the public against the contamination of shell-fish.

In most directions the tendency to the pollution of our water supplies increases with the demand for pure water, and the area from which such water can be obtained in the neighbourhood of our towns is diminishing. The existing local authorities have conflicting interests when dealing with river pollution, and considerations of guarding the purity of streams are often subordinated to those of refuse disposal and manufacturing requirements. What too often happens is that a sanitary authority, situated toward the head of the stream or upon one of its tributaries, 